Research Article

\title{
Optimality Conditions for Infinite Order Distributed Parabolic Systems with Multiple Time Delays Given in Integral Form
}

\author{
Bahaa G. M. ${ }^{1,2}$ \\ ${ }^{1}$ Department of Mathematics, Faculty of Science, Taibah University, \\ Al-Madinah Al-Munawwarah, Saudi Arabia \\ ${ }^{2}$ Department of Mathematics, Faculty of Science, Beni-Suef University, Beni-Suef, Egypt
}

Correspondence should be addressed to G. M. Bahaa, bahaa_gm@hotmail.com

Received 1 March 2012; Accepted 29 April 2012

Academic Editor: Weihai Zhang

Copyright (C 2012 Bahaa G. M.. This is an open access article distributed under the Creative Commons Attribution License, which permits unrestricted use, distribution, and reproduction in any medium, provided the original work is properly cited.

The optimal boundary control problem for $(n \times n)$ infinite order distributed parabolic systems with multiple time delays given in the integral form both in the state equations and in the Neumann boundary conditions is considered. Constraints on controls are imposed. Necessary and suffacient optimality conditions for the Neumann problem with the quadratic performance functional are derived.

\section{Introduction}

Distributed parameters systems with delays can be used to describe many phenomena in the real world. As is well known, heat conduction, properties of elastic-plastic material, fluid dynamics, diffusion-reaction processes, the transmission of the signals at a certain distance by using electric long lines, and so forth, all lie within this area. The object that we are studying (temperature, displacement, concentration, velocity, etc.) is usually referred to as the state.

During the last twenty years, equations with deviating argument have been applied not only in applied mathematics, physics, and automatic control, but also in some problems of economy and biology. Currently, the theory of equations with deviating arguments constitutes a very important subfield of mathematical control theory.

Consequently, equations with deviating arguments are widely applied in optimal control problems of distributed parameter system with time delays [1].

The optimal control problems of distributed parabolic systems with time-delayed boundary conditions have been widely discussed in many papers and monographs. A fundamental study of such problems is given by [2] and was next developed by [3, 4]. It was 
also intensively investigated by $[1,5-16]$ in which linear quadratic problem for parabolic systems with time delays given in the different form (constant, time delays, time-varying delays, time delays given in the integral form, etc.) was presented.

The necessary and sufficient conditions of optimality for systems consist of only one equation and for $(n \times n)$ systems governed by different types of partial differential equations defined on spaces of functions of infinitely many variables and also for infinite order systems are discussed for example in $[9,11,15-18]$ in which the argument of $[19,20]$ was used.

Making use of the Dubovitskii-Milyutin Theorem in [13, 21-28] the necessary and sufficient conditions of optimality for similar systems governed by second order operator with an infinite number of variables and also for infinite order systems were investigated. The interest in the study of this class of operators is stimulated by problems in quantum field theory.

In particular, the papers of $[1,8]$ present necessary and sufficient optimality conditions for the Neumann problem with quadratic performance functionals, applied to a single one equation of second-order parabolic system with fixed time delay and with multiple time delays given in the integral form both in the state equations and in the Neumann boundary conditions, respectively. Such systems constitute a more complex case of distributed parameter systems with time delays given in the integral form.

Also in $[9,11]$ time-optimal boundary control for a single one equation distributed infinite order parabolic and hyperbolic systems in which constant time lags appear in the integral form both in the state equation and in the Neumann boundary condition is present. Some specific properties of the optimal control are discussed.

In this paper we recall the problem in a more general formulation. A distributed parameter for infinite order parabolic $(n \times n)$ systems with multiple time delays given in the integral form both in the state equations and in the Neumann boundary conditions is considered. Such an infinite order parabolic system can be treated as a generalization of the mathematical model for a plasma control process. The quadratic performance functionals defined over a fixed time horizon are taken and some constraints are imposed on the initial state and the boundary control. Such a system may be viewed as a linear representation of many diffusion processes, in which time-delayed signals are introduced at a spatial boundary, and there is a freedom in choosing the controlled process initial state. Following a line of the Lions scheme, necessary and sufficient optimality conditions for the Neumann problem applied to the above system were derived. The optimal control is characterized by the adjoint equations.

This paper is organized as follows. In Section 1, we introduce spaces of functions of infinite order. In Section 2, we formulate the mixed Neumann problem for infinite order parabolic operator with multiple time delays given in the integral form. In Section 3, the boundary optimal control problem for this case is formulated, then we give the necessary and sufficient conditions for the control to be an optimal. In Section 4, we generalized the discussion to two cases, the first case: the optimal control for $(2 \times 2)$ coupled infinite order parabolic systems is studied. The second case: the optimal control for $(n \times n)$ coupled infinite order parabolic systems was to be formulated.

\section{Sobolev Spaces with Infinite Order}

The object of this section is to give the definition of some function spaces of infinite order and the chains of the constructed spaces which will be used later. 
Let $\Omega$ be a bounded open set of $\mathbb{R}^{n}$ with a smooth boundary $\Gamma$, which is a $C^{\infty}$ manifold of dimension $(n-1)$. Locally, $\Omega$ is totally on one side of $\Gamma$. We define the infinite order Sobolev space $W^{\infty}\left\{a_{\alpha}, 2\right\}(\Omega)$ of infinite order of periodic functions $\phi(x)$ defined on $\Omega$ [29-31] as follows:

$$
W^{\infty}\left\{a_{\alpha}, 2\right\}(\Omega)=\left\{\phi(x) \in \mathrm{C}^{\infty}(\Omega): \sum_{|\alpha|=0}^{\infty} a_{\alpha}\left\|\mathrm{D}^{\alpha} \phi\right\|_{2}^{2}<\infty\right\},
$$

where $C^{\infty}(\Omega)$ is the space of infinitely differentiable functions, $a_{\alpha} \geq 0$ is a numerical sequence, and $\|\cdot\|_{2}$ is the canonical norm in the space $L^{2}(\Omega)$, and

$$
D^{\alpha}=\frac{\partial^{|\alpha|}}{\left(\partial x_{1}\right)^{\alpha_{1}} \cdots\left(\partial x_{n}\right)^{\alpha_{n}}}
$$

$\alpha=\left(\alpha_{1}, \ldots, \alpha_{n}\right)$ being a multi-index for differentiation, $|\alpha|=\sum_{i=1}^{n} \alpha_{i}$.

The space $W^{-\infty}\left\{a_{\alpha}, 2\right\}(\Omega)$ is defined as the formal conjugate space to the space $W^{\infty}\left\{a_{\alpha}, 2\right\}(\Omega)$, namely:

$$
W^{-\infty}\left\{a_{\alpha}, 2\right\}(\Omega)=\left\{\psi(x): \psi(x)=\sum_{|\alpha|=0}^{\infty}(-1)^{|\alpha|} a_{\alpha} D^{\alpha} \psi_{\alpha}(x)\right\},
$$

where $\psi_{\alpha} \in L^{2}(\Omega)$ and $\sum_{|\alpha|=0}^{\infty} a_{\alpha}\left\|\psi_{\alpha}\right\|_{2}^{2}<\infty$. the formula:

The duality pairing of the spaces $W^{\infty}\left\{a_{\alpha}, 2\right\}(\Omega)$ and $W^{-\infty}\left\{a_{\alpha}, 2\right\}(\Omega)$ is postulated by

$$
(\phi, \psi)=\sum_{|\alpha|=0}^{\infty} a_{\alpha} \int_{\Omega} \psi_{\alpha}(x) D^{\alpha} \phi(x) d x,
$$

where

$$
\phi \in W^{\infty}\left\{a_{\alpha}, 2\right\}(\Omega), \quad \psi \in W^{-\infty}\left\{a_{\alpha}, 2\right\}(\Omega) .
$$

From above, $W^{\infty}\left\{a_{\alpha}, 2\right\}(\Omega)$ is everywhere dense in $L^{2}(\Omega)$ with topological inclusions and $W^{-\infty}\left\{a_{\alpha}, 2\right\}(\Omega)$ denotes the topological dual space with respect to $L^{2}(\Omega)$, so we have the following chain of inclusions:

$$
W^{\infty}\left\{a_{\alpha}, 2\right\}(\Omega) \subseteq L^{2}(\Omega) \subseteq W^{-\infty}\left\{a_{\alpha}, 2\right\}(\Omega) .
$$


We now introduce $L^{2}\left(0, T ; L^{2}(\Omega)\right)$ which we will denoted by $L^{2}(Q)$, where $\left.Q=\Omega \times\right] 0, T[$ denotes the space of measurable functions $t \rightarrow \phi(t)$ such that

$$
\|\phi\|_{L^{2}(Q)}=\left(\int_{0}^{T}\|\phi(t)\|_{2}^{2} d t\right)^{1 / 2}<\infty
$$

endowed with the scalar product $(f, g)=\int_{0}^{T}(f(t), g(t))_{L^{2}(\Omega)} d t, L^{2}(Q)$ is a Hilbert space.

In the same manner we define the spaces $L^{2}\left(0, T ; W^{\infty}\left\{a_{\alpha}, 2\right\}(\Omega)\right)$, and $L^{2}(0, T$; $\left.W^{-\infty}\left\{a_{\alpha}, 2\right\}(\Omega)\right)$, as its formal conjugate.

Also, we have the following chain of inclusions:

$$
L^{2}\left(0, T ; W^{\infty}\left\{a_{\alpha}, 2\right\}(\Omega)\right) \subseteq L^{2}(Q) \subseteq L^{2}\left(0, T ; W^{-\infty}\left\{a_{\alpha}, 2\right\}(\Omega)\right)
$$

The construction of the Cartesian product of $n$-times to the above Hilbert spaces can be constructed, for example

$$
\begin{aligned}
\left(W^{\infty}\left\{a_{\alpha}, 2\right\}(\Omega)\right)^{n} & =\underbrace{W^{\infty}\left\{a_{\alpha}, 2\right\}(\Omega) \times W^{\infty}\left\{a_{\alpha}, 2\right\}(\Omega) \times \cdots \times W^{\infty}\left\{a_{\alpha}, 2\right\}(\Omega)}_{n \text {-times }} \\
& =\prod_{i=1}^{n}\left(W^{\infty}\left\{a_{\alpha}, 2\right\}(\Omega)\right)^{i},
\end{aligned}
$$

with norm defined by:

$$
\|\phi\|_{\left(W^{\infty}\left\{a_{\alpha}, 2\right\}(\Omega)\right)^{n}}=\sum_{i=1}^{n}\left\|\phi_{i}\right\|_{W^{\infty}\left\{a_{\alpha, 2}\right\}(\Omega)^{\prime}}
$$

where $\phi=\left(\phi_{1}, \phi_{2}, \ldots, \phi_{n}\right)=\left(\phi_{i}\right)_{i=1}^{n}$ is a vector function and $\phi_{i} \in W^{\infty}\left\{a_{\alpha}, 2\right\}(\Omega)$.

Finally, we have the following chain of inclusions:

$$
\left(L^{2}\left(0, T ; W^{\infty}\left\{a_{\alpha}, 2\right\}(\Omega)\right)\right)^{n} \subseteq\left(L^{2}(Q)\right)^{n} \subseteq\left(L^{2}\left(0, T ; W^{-\infty}\left\{a_{\alpha}, 2\right\}(\Omega)\right)\right)^{n}
$$

where $\left(L^{2}\left(0, T ; W^{-\infty}\left\{a_{\alpha}, 2\right\}(\Omega)\right)\right)^{n}$ are the dual spaces of $\left(L^{2}\left(0, T ; W^{\infty}\left\{a_{\alpha}, 2\right\}(\Omega)\right)\right)^{n}$. The spaces considered in this paper are assumed to be real. 


\section{Mixed Neumann Problem for Infinite Order Parabolic System with Multiple Time Lags}

The object of this section is to formulate the following mixed initial boundary value Neumann problem for infinite order parabolic system with multiple time delays which defines the state of the system model $[1,5-11,18,24,26]$.

$$
\begin{gathered}
\frac{\partial y}{\partial t}+\mathcal{A}(t) y(x, t)+\sum_{i=1}^{m} \int_{a_{i}}^{b_{i}} b_{i}(x, t) y\left(x, t-h_{i}\right) d h_{i}=u, \\
(x, t) \in \Omega \times(0, T), \quad h_{i} \in\left(a_{i}, b_{i}\right) \\
y\left(x, t^{\prime}\right)=\Phi_{0}\left(x, t^{\prime}\right), \quad\left(x, t^{\prime}\right) \in \Omega \times(-\Delta, 0), \\
y(x, 0)=y_{0}(x), \quad x \in \Omega, \\
\frac{\partial y}{\partial v_{A}}(x, t)=\sum_{s=1}^{l} \int_{c_{s}}^{d_{s}} c_{S}(x, t) y\left(x, t-k_{s}\right) d k_{s}+v, \quad(x, t) \in \Gamma \times(0, T), k_{s} \in\left(c_{s}, d_{s}\right), \\
y\left(x, t^{\prime}\right)=\Psi_{0}\left(x, t^{\prime}\right), \quad\left(x, t^{\prime}\right) \in \Gamma \times(-\Delta, 0)
\end{gathered}
$$

where $\Omega \subset R^{n}$ has the same properties as in Section 1 . We have

$$
\begin{gathered}
y \equiv y(x, t ; u), \quad y(0) \equiv y(x, 0 ; u), \quad y(T) \equiv y(x, T ; u), \quad u \equiv u(x, t), \quad v \equiv v(x, t), \\
Q=\Omega \times(0, T), \quad \bar{Q}=\bar{\Omega} \times[0, T], \quad Q_{0}=\Omega \times[-\Delta, 0), \quad \Sigma=\Gamma \times(0, T), \quad \Sigma_{0}=\Gamma \times[-\Delta, 0),
\end{gathered}
$$

(i) $T$ is a specified positive number representing a finite time horizon,

(ii) $h_{i}, k_{s}$ are time delays, such that $h_{i} \in\left(a_{i}, b_{i}\right)$ and $k_{s} \in\left(c_{s}, d_{s}\right)$ where $0<a_{1}<a_{2}<$ $\cdots<a_{m}, 0<b_{1}<b_{2}<\cdots<b_{m}$, for $i=1,2, \ldots, m$ and $0<c_{1}<c_{2}<\cdots<c_{l}$, $0<d_{1}<d_{2}<\cdots<d_{l}$, for $s=1,2, \ldots, l$,

(iii) $b_{i}(t), i=1,2, \ldots, m$ are given real $C^{\infty}$ functions defined on $\bar{Q}$,

(iv) $c_{s}(x, t), s=1,2, \ldots, l$ are given real $C^{\infty}$ functions defined on $\Sigma$,

(v) $\Delta=\max \left\{b_{m}, d_{l}\right\}$,

(vi) $y$ is a function defined on $Q$ such that $\Omega \times(0, T) \ni(x, t) \rightarrow y(x, t) \in R$,

(vii) $u, v$ are functions defined on $Q$ and $\Sigma$ such that $\Omega \times(0, T) \ni(x, t) \rightarrow u(x, t) \in R$ and $\Gamma \times(0, T) \ni(x, t) \rightarrow v(x, t) \in R$,

(viii) $\Phi_{0}, \Psi_{0}$ are initial functions defined on $Q_{0}$ and $\Sigma_{0}$, respectively, such that $\Omega \times[-\Delta, 0) \ni$ $\left(x, t^{\prime}\right) \rightarrow \Phi_{0}\left(x, t^{\prime}\right) \in R . \Gamma \times[-\Delta, 0) \ni\left(x, t^{\prime}\right) \rightarrow \Psi_{0}\left(x, t^{\prime}\right) \in R$. 
The parabolic operator $(\partial / \partial t)+\mathcal{A}(t)$ in the state equation (3.1) is an infinite order parabolic operator and $\mathcal{A}(t)[17,21,29-31]$ is given by:

$$
\begin{gathered}
\mathcal{A} y=\sum_{|\alpha|=0}^{\infty}(-1)^{|\alpha|} a_{\alpha} D^{2 \alpha} y(x, t), \\
\mathcal{A}=\sum_{|\alpha|=0}^{\infty}(-1)^{|\alpha|} a_{\alpha} D^{2 \alpha}
\end{gathered}
$$

is an infinite order self-adjoint elliptic partial differential operator maps $W^{\infty}\left\{a_{\alpha}, 2\right\}(\Omega)$ onto $W^{-\infty}\left\{a_{\alpha}, 2\right\}(\Omega)$.

For this operator we define the bilinear form as follows.

Definition 3.1. For each $t \in(0, T)$, we define a family of bilinear forms on $W^{\infty}\left\{a_{\alpha}, 2\right\}(\Omega)$ by:

$$
\pi(t ; y, \phi)=(\mathcal{A}(t) y, \phi)_{L^{2}(\Omega)}, \quad y, \phi \in W^{\infty}\left\{a_{\alpha}, 2\right\}(\Omega),
$$

where $\mathcal{A}(t)$ maps $W^{\infty}\left\{a_{\alpha}, 2\right\}(\Omega)$ onto $W^{-\infty}\left\{a_{\alpha}, 2\right\}(\Omega)$ and takes the above form. Then

$$
\begin{aligned}
\pi(t ; y, \phi) & =(\mathcal{A}(t) y, \phi)_{L^{2}(\Omega)} \\
& =\left(\sum_{|\alpha|=0}^{\infty}(-1)^{|\alpha|} a_{\alpha} D^{2 \alpha} y(x, t), \phi(x)\right)_{L^{2}(\Omega)} \\
& =\int_{\Omega} \sum_{|\alpha|=0}^{\infty} a_{\alpha} D^{\alpha} y(x) D^{\alpha} \phi(x) d x .
\end{aligned}
$$

Lemma 3.2. The bilinear form $\pi(t ; y, \phi)$ is coercive on $W^{\infty}\left\{a_{\alpha}, 2\right\}(\Omega)$, that is,

$$
\pi(t ; y, y) \geq \lambda\|y\|_{W^{\infty}\left\{a_{\alpha}, 2\right\}(\Omega)^{\prime}}^{2} \quad \lambda>0 .
$$

Proof. It is well known that the ellipticity of $\mathcal{A}(t)$ is sufficient for the coerciveness of $\pi(t ; y, \phi)$ on $W^{\infty}\left\{a_{\alpha}, 2\right\}(\Omega)$ :

$$
\pi(t ; \phi, \psi)=\int_{\Omega} \sum_{|\alpha|=0}^{\infty} a_{\alpha} D^{\alpha} \phi D^{\alpha} \psi d x .
$$


Then

$$
\begin{aligned}
\pi(t ; y, y) & =\int_{\Omega} \sum_{|\alpha|=0}^{\infty} a_{\alpha} D^{\alpha} y D^{\alpha} y d x \\
& \geq \sum_{|\alpha|=0}^{\infty} a_{\alpha}\left\|D^{2 \alpha} y(x)\right\|_{L^{2}(\Omega)}^{2} \\
& \geq \lambda\|y\|_{W^{\infty}\left\{a_{\alpha}, 2\right\}(\Omega)}^{2} \quad \lambda>0 .
\end{aligned}
$$

Also we have

$$
\begin{aligned}
& \forall y, \phi \in W^{\infty}\left\{a_{\alpha}, 2\right\}(\Omega) \text { the function } t \longrightarrow \pi(t ; y, \phi) \\
& \text { is continuously differentiable in }(0, T) \text { and } \pi(t ; y, \phi)=\pi(t ; \phi, y) .
\end{aligned}
$$

Equations (3.1)-(3.5) constitute a Neumann problem. Then the left-hand side of the boundary condition (3.4) may be written in the following form:

$$
\frac{\partial y(x, t)}{\partial v_{\mathcal{A}}}=\sum_{|\omega|=0}^{\infty}\left(D^{\omega} y(x, t)\right) \cos \left(n, x_{k}\right)=q(x, t), \quad x \in \Gamma, t \in(0, T)
$$

where $\partial / \partial v_{\mathcal{A}}$ is a normal derivative at $\Gamma$, directed towards the exterior of $\Omega$, and $\cos \left(n, x_{k}\right)$ is the $k$ th direction cosine of $n$, with $n$ being the normal at $\Gamma$ exterior to $\Omega$.

Then (3.4) can be written as:

$$
q(x, t)=\sum_{s=1}^{l} \int_{c_{s}}^{d_{s}} c_{s}(x, t) y\left(x, t-k_{s}\right) d k_{s}+v(x, t), \quad x \in \Gamma, t \in(0, T) .
$$

Remark 3.3. We will apply the indication $q(x, t)$ appearing in (3.14) to prove the existence of a unique solution for (3.1)-(3.5).

We will formulate sufficient conditions for the existence of a unique solution of the mixed boundary value problem (3.1)-(3.5) for the cases where the boundary control $v \in$ $L^{2}(\Sigma)$. by:

For this purpose, we introduce the Sobolev space $W^{\infty, 1}(Q)$ [20, Vol. 2, page 6] defined

$$
W^{\infty, 1}(Q)=L^{2}\left(0, T ; W^{\infty}\left\{a_{\alpha}, 2\right\}(\Omega)\right) \cap W^{1}\left(0, T ; L^{2}(\Omega)\right),
$$


which is a Hilbert space normed by

$$
\begin{aligned}
\|y\|_{W^{\infty, 1}(Q)} & =\left[\int_{0}^{T} \int\|y\|_{W^{\infty}\left\{a_{\alpha}, 2\right\}(\Omega)}^{2} d t+\|y\|_{W^{1}\left(0, T ; L^{2}(\Omega)\right)}^{2}\right]^{1 / 2} \\
& =\left[\int_{Q}\left(\sum_{|\alpha|=0}^{\infty} a_{\alpha}\left|D^{\alpha} y\right|^{2}+\left|\frac{\partial y}{\partial t}\right|^{2}\right) d x d t\right]^{1 / 2} \\
& =\left[\int_{Q}\left(a_{0}|y|^{2}+\sum_{|\alpha|=1}^{\infty} a_{\alpha}\left|D^{\alpha} y\right|^{2}+\left|\frac{\partial y}{\partial t}\right|^{2}\right) d x d t\right]^{1 / 2}, \quad a_{0}>0,
\end{aligned}
$$

where the space $W^{1}\left(0, T ; L^{2}(\Omega)\right)$ denotes the Sobolev space of order 1 of functions defined on $(0, T)$ and taking values in $L^{2}(\Omega)$ [20, Vol. 1].

The existence of a unique solution for the mixed initial-boundary value problem (3.1)-(3.5) on the cylinder $Q$ can be proved using a constructive method, that is, solving at first equations (3.1)-(3.5) on the subcylinder $Q_{1}$ and in turn on $Q_{2}$ and so forth, until the procedure covers the whole cylinder $Q$. In this way, the solution in the previous step determines the next one.

For simplicity, we introduce the following notation:

$$
E_{j}=((j-1) \lambda, j \lambda), \quad Q_{j}=\Omega \times E_{j}, \quad \Sigma_{j}=\Gamma \times E_{j} \quad \text { for } j=1, \ldots, K, \lambda=\min \left\{a_{1}, c_{1}\right\} .
$$

Making use of the results of $[7,20]$ we can prove that the following result holds.

Theorem 3.4. Let $y_{0}, \Phi_{0}, \Psi_{0}, v$ and $u$ be given with $y_{0} \in W^{\infty}\left\{a_{\alpha}, 2\right\}(\Omega), \Phi_{0} \in W^{\infty, 1}\left(Q_{0}\right), \Psi_{0} \in$ $L^{2}\left(\Sigma_{0}\right), v \in L^{2}(\Sigma)$ and $u \in W^{-\infty,-1}(Q)$. Then, there exists a unique solution $y \in W^{\infty, 1}(Q)$ for the mixed initial-boundary value problem (3.1)-(3.5). Moreover, $y(\cdot, j \lambda) \in W^{\infty}\left\{a_{\alpha}, 2\right\}(\Omega)$ for $j=$ $1, \ldots, K$.

\section{Problem Formulation-Optimization Theorems}

Now, we formulate the optimal control problem for (3.1)-(3.5) in the context of the Theorem 3.4, that is $v \in L^{2}(\Sigma)$. problem.

Let us denote by $U=L^{2}(\Sigma)$ the space of controls. The time horizon $T$ is fixed in our

The performance functional is given by

$$
I(v)=\lambda_{1} \int_{Q}\left[y(x, t ; v)-z_{d}\right]^{2} d x d t+\lambda_{2} \int_{\Sigma}(N v) v d \Gamma d t
$$

where $\lambda_{i} \geq 0$, and $\lambda_{1}+\lambda_{2}>0, z_{d}$ is a given element in $L^{2}(Q) ; N$ is a positive linear operator on $L^{2}(\Sigma)$ into $L^{2}(\Sigma)$. 


\section{Control Contraints}

We define the set of admissible controls $U_{\text {ad }}$ such that

$$
U_{\mathrm{ad}} \text { is closed, convex subset of } U=L^{2}(\Sigma) \text {. }
$$

Let $y(x, t ; v)$ denote the solution of the mixed initial-boundary value problem (3.1)(3.5) at $(x, t)$ corresponding to a given control $v \in U_{\text {ad }}$. We note from Theorem 3.4 that for any $v \in U_{\text {ad }}$ the performance functional (4.1) is well-defined since $(v) \in W^{\infty, 1}(Q) \subset L^{2}(Q)$.

Making use of the Loins's scheme we will derive the necessary and sufficient conditions of optimality for the optimization problem (3.1)-(3.5), (4.1), (4.2). The solving of the formulated optimal control problem is equivalent to seeking a $v^{*} \in U_{\text {ad }}$ such that

$$
I\left(v^{*}\right) \leq I(v), \quad \forall v \in U_{\mathrm{ad}}
$$

From the Lion's scheme [19, Theorem 1.3, page 10], it follows that for $\lambda_{2}>0$ a unique optimal control $v^{*}$ exists. Moreover, $v^{*}$ is characterized by the following condition:

$$
I^{\prime}\left(v^{*}\right)\left(v-v^{*}\right) \geq 0, \quad \forall v \in U_{\mathrm{ad}} .
$$

For the performance functional of form (4.1) the relation (4.4) can be expressed as

$$
\lambda_{1} \int_{Q}\left(y\left(v^{*}\right)-z_{d}\right)\left[y(v)-y\left(v^{*}\right)\right] d x d t+\lambda_{2} \int_{\Sigma} N v^{*}\left(v-v^{*}\right) d \Gamma d t \geq 0, \quad \forall v \in U_{\mathrm{ad}}
$$

In order to simplify (4.5), we introduce the adjoint equation, and for every $v \in U_{\mathrm{ad}}$, we define the adjoint variable $p=p(v) \equiv p(x, t ; v)$ as the solution of the equations:

$$
\begin{gathered}
\frac{-\partial p(v)}{\partial t}+\mathcal{A}^{*}(t) p(v)+\sum_{i=1}^{m} \int_{a_{i}}^{b_{i}} b_{i}\left(x, t+h_{i}\right) p\left(x, t+h_{i} ; v\right) d h_{i}=\lambda_{1}\left(y(v)-z_{d}\right), \\
\quad(x, t) \in \Omega \times(0, T-\Delta), \quad h_{i} \in\left(a_{i}, b_{i}\right), \\
\frac{-\partial p(v)}{\partial t}+\mathcal{A}^{*}(t) p(v)=\lambda_{1}\left(y(v)-z_{d}\right), \quad(x, t) \in \Omega \times(T-\Delta, T), \\
p(x, T ; v)=0, \quad x \in \Omega, \\
\frac{\partial p(v)}{\partial v_{\mathcal{A}^{*}}}(x, t)=\sum_{s=1}^{l} \int_{c_{S}}^{d_{s}} c_{S}\left(x, t+k_{s}\right) p\left(x, t+k_{s} ; v\right) d k_{s}, \quad(x, t) \in \Gamma \times(0, T-\Delta(T)), k_{s} \in\left(c_{s}, d_{s}\right), \\
\frac{\partial p(v)}{\partial v_{\mathbb{A}^{*}}}(x, t)=0, \quad(x, t) \in \Gamma \times(T-\Delta(T), T),
\end{gathered}
$$


where

$$
\begin{aligned}
& \frac{\partial p(v)}{\partial \mathcal{v}_{\mathcal{A}^{*}}}(x, t)=\sum_{|\omega|=0}^{\infty}\left(D^{\omega} p(v)\right) \cos \left(n, x_{\omega}\right)(x, t), \\
& \mathcal{A}^{*}(t) p(v)=\sum_{|\alpha|=0}^{\infty}(-1)^{|\alpha|} a_{\alpha} D^{2 \alpha} p(x, t) .
\end{aligned}
$$

As in the above section with change of variables, that is, with reversed sense of time. that is, $t^{\prime}=T-t$, for given $z_{d} \in L^{2}(Q)$ and any $v \in L^{2}(\Sigma)$, there exists a unique solution $p(v) \in W^{\infty, 1}(Q)$ for problem (4.6)-(4.11).

The existence of a unique solution for the problem (4.6)-(4.11) on the cylinder $\Omega \times$ $(0, T)$ can be proved using a constructive method. It is easy to notice that for given $z_{d}$ and $u$, the problem (4.6)-(4.11) can be solved backwards in time starting from $t=T$, that is, first solving (4.6)-(4.11) on the subcylinder $Q_{K}$ and in turn on $Q_{K-1}$, and so forth until the procedure covers the whole cylinder $\Omega \times(0, T)$. For this purpose, we may apply Theorem 3.4 (with an obvious change of variables).

Hence, using Theorem 3.4, the following result can be proved.

Lemma 4.1. Let the hypothesis of Theorem 3.4 be satisfied. Then for given $z_{d} \in L^{2}\left(\Omega, R^{\infty}\right)$ and any $v \in L^{2}(\Sigma)$, there exists a unique solution $p(v) \in W^{\infty, 1}(Q)$ for the adjoint problem (4.6)-(4.11).

We simplify (4.5) using the adjoint equation (4.6)-(4.11). For this purpose denoting by $p(0) \equiv p(x, 0 ; v)$ and $p(T) \equiv p(x, T ; v)$, respectively, setting $v=v^{*}$ in (4.6)-(4.11), multiplying both sides of (4.6) and (4.7) by $y(v)-y\left(v^{*}\right)$, then integrating over $\Omega \times(0, T-\Delta)$ and $\Omega \times(T-$ $\Delta, T)$, respectively and then adding both sides of $(4.6),(4.11)$, we get

$$
\begin{aligned}
\lambda_{1} \int_{Q}( & \left.y\left(v^{*}\right)-z_{d}\right)\left[y(v)-y\left(v^{*}\right)\right] d x d t \\
= & \int_{Q}\left(-\frac{\partial p\left(v^{*}\right)}{\partial t}+\mathcal{A}^{*}(t) p\left(v^{*}\right)\right) \times\left[y(v)-y\left(v^{*}\right)\right] d x d t \\
& +\int_{0}^{T-\Delta} \int_{\Omega}\left(\sum_{i=1}^{m} \int_{a_{i}}^{b_{i}} b_{i}\left(x, t+h_{i}\right) p\left(x, t+h_{i} ; v^{*}\right) d h_{i}\right) \times\left[y(x, t ; v)-y\left(x, t ; v^{*}\right)\right] d x d t \\
= & \int_{0}^{T} \int_{\Omega} p\left(v^{*}\right) \frac{\partial}{\partial t}\left[y(v)-y\left(v^{*}\right)\right] d x d t \\
& +\int_{0}^{T} \int_{\Omega} A^{*}(t) p\left(v^{*}\right)\left[y(v)-y\left(v^{*}\right)\right] d x d t \\
& +\sum_{i=1}^{m} \int_{a_{i}}^{b_{i}} \int_{\Omega} \int_{0}^{T-\Delta}\left(b_{i}\left(x, t+h_{i}\right) p\left(x, t+h_{i} ; v^{*}\right)\right) \times\left[y(x, t ; v)-y\left(x, t ; v^{*}\right)\right] d x d t d h_{i} .
\end{aligned}
$$


Using (3.1), the first integral on the right-hand side of (4.13) can be written as:

$$
\begin{aligned}
& \int_{0}^{T} \int_{\Omega} p\left(v^{*}\right) \frac{\partial}{\partial t}\left[y(v)-y\left(v^{*}\right)\right] d x d t \\
& =-\int_{Q} p\left(v^{*}\right) \mathcal{A}(t)\left(y(v)-y\left(v^{*}\right)\right) d x d t \\
& -\sum_{i=1}^{m} \int_{a_{i}}^{b_{i}} \int_{\Omega} \int_{0}^{T} p\left(x, t ; v^{*}\right) b_{i}(x, t) \times\left[y\left(x, t-h_{i} ; v\right)-y\left(x, t-h_{i} ; v^{*}\right)\right] d t d x d h_{i} \\
& =-\int_{Q} p\left(v^{*}\right) \mathcal{A}(t)\left(y(v)-y\left(v^{*}\right)\right) d x d t \\
& -\sum_{i=1}^{m} \int_{a_{i}}^{b_{i}} \int_{\Omega} \int_{-h_{i}}^{T-h_{i}} p\left(x, t^{\prime}+h_{i} ; v^{*}\right) b_{i}\left(x, t^{\prime}+h_{i}\right) \times\left[y\left(x, t^{\prime} ; v\right)-y\left(x, t^{\prime} ; v^{*}\right)\right] d t^{\prime} d x d h_{i} \\
& =-\int_{\Omega} p\left(v^{*}\right) \mathcal{A}(t)\left(y(v)-y\left(v^{*}\right)\right) d x d t \\
& -\sum_{i=1}^{m} \int_{a_{i}}^{b_{i}} \int_{\Omega} \int_{-h_{i}}^{0} p\left(x, t^{\prime}+h_{i} ; v^{*}\right) b_{i}\left(x, t^{\prime}+h_{i}\right) \times\left[y\left(x, t^{\prime} ; v\right)-y\left(x, t^{\prime} ; v^{*}\right)\right] d t^{\prime} d x d h_{i} \\
& -\sum_{i=1}^{m} \int_{a_{i}}^{b_{i}} \int_{\Omega} \int_{0}^{T-\Delta} p\left(x, t^{\prime}+h_{i} ; v^{*}\right) b_{i}\left(x, t^{\prime}+h_{i}\right) \times\left[y\left(x, t^{\prime} ; v\right)-y\left(x, t^{\prime} ; v^{*}\right)\right] d t^{\prime} d x d h_{i} \\
& -\sum_{i=1}^{m} \int_{a_{i}}^{b_{i}} \int_{\Omega} \int_{T-\Delta}^{T-h_{i}} p\left(x, t^{\prime}+h_{i} ; v^{*}\right) b_{i}\left(x, t^{\prime}+h_{i}\right) \times\left[y\left(x, t^{\prime} ; v\right)-y\left(x, t^{\prime} ; v^{*}\right)\right] d t^{\prime} d x d h_{i} \\
& =-\int_{Q} p\left(v^{*}\right) \mathcal{A}(t)\left(y(v)-y\left(v^{*}\right)\right) d x d t \\
& -\sum_{i=1}^{m} \int_{a_{i}}^{b_{i}} \int_{\Omega} \int_{-h_{i}}^{0} p\left(x, t^{\prime}+h_{i} ; v^{*}\right) b_{i}\left(x, t^{\prime}+h_{i}\right) \times\left[y\left(x, t^{\prime} ; v\right)-y\left(x, t^{\prime} ; v^{*}\right)\right] d t^{\prime} d x d h_{i} \\
& -\sum_{i=1}^{m} \int_{a_{i}}^{b_{i}} \int_{\Omega} \int_{0}^{T-\Delta} p\left(x, t^{\prime}+h_{i} ; v^{*}\right) b_{i}\left(x, t^{\prime}+h_{i}\right) \times\left[y\left(x, t^{\prime} ; v\right)-y\left(x, t^{\prime} ; v^{*}\right)\right] d t^{\prime} d x d h_{i} \\
& -\sum_{i=1}^{m} \int_{a_{i}}^{b_{i}} \int_{\Omega} \int_{T-\Delta+h_{i}}^{T} p\left(x, t ; v^{*}\right) b_{i}(x, t) \times\left[y\left(x, t-h_{i} ; v\right)-y\left(x, t-h_{i} ; v^{*}\right)\right] d t d x d h_{i} .
\end{aligned}
$$

Using Green's formula, the second integral on the right-hand side of (4.13) can be written as:

$$
\begin{aligned}
\int_{0}^{T} \int_{\Omega} \mathcal{A}^{*}(t) p\left(v^{*}\right)\left[y(v)-y\left(v^{*}\right)\right] d x d t \\
\quad=\int_{0}^{T} \int_{\Omega} p\left(v^{*}\right) \mathcal{A}(t)\left[y(v)-y\left(v^{*}\right)\right] d x d t
\end{aligned}
$$




$$
\begin{aligned}
& +\int_{0}^{T} \int_{\Gamma} p\left(v^{*}\right)\left(\frac{\partial y(v)}{\partial v_{A}}-\frac{\partial y\left(v^{*}\right)}{\partial v_{A}}\right) d \Gamma d t \\
& -\int_{0}^{T} \int_{\Gamma} \frac{\partial p\left(v^{*}\right)}{\partial v_{A^{*}}}\left[y(v)-y\left(v^{*}\right)\right] d \Gamma d t .
\end{aligned}
$$

Using the boundary condition (3.2), one can transform the second integral on the right-hand side of (4.15) into the form:

$$
\begin{aligned}
& \int_{0}^{T} \int_{\Gamma} p\left(v^{*}\right)\left(\frac{\partial y(v)}{\partial v_{A}}-\frac{\partial y\left(v^{*}\right)}{\partial v_{A}}\right) d \Gamma d t \\
& =\sum_{s=1}^{l} \int_{c_{s}}^{d_{s}} \int_{\Gamma} \int_{0}^{T} p\left(x, t ; v^{*}\right) c_{s}(x, t) \times\left[y\left(x, t-k_{s} ; v\right)-y\left(x, t-k_{s} ; v^{*}\right)\right] d \Gamma d t d k_{s} \\
& +\int_{0}^{T} \int_{\Gamma} p\left(v^{*}\right)\left(v-v^{*}\right) d \Gamma d t \\
& =\sum_{s=1}^{l} \int_{c_{s}}^{d_{s}} \int_{\Gamma} \int_{-k_{s}}^{T-k_{s}} p\left(x, t^{\prime}+k_{s} ; v^{*}\right) c_{s}\left(x, t^{\prime}+k_{s}\right) \times\left[y\left(x, t^{\prime} ; v\right)-y\left(x, t^{\prime} ; v^{*}\right)\right] d t^{\prime} d \Gamma d k_{s} \\
& +\int_{0}^{T} \int_{\Gamma} p\left(v^{*}\right)\left(v-v^{*}\right) d \Gamma d t \\
& =\sum_{s=1}^{l} \int_{c_{s}}^{d_{s}} \int_{\Gamma} \int_{-k_{s}}^{0} p\left(x, t^{\prime}+k_{s} ; v^{*}\right) c_{s}\left(x, t^{\prime}+k_{s}\right) \times\left[y\left(x, t^{\prime} ; v\right)-y\left(x, t^{\prime} ; v^{*}\right)\right] d t^{\prime} d \Gamma d k_{s} \\
& +\sum_{s=1}^{l} \int_{c_{s}}^{d_{s}} \int_{\Gamma} \int_{0}^{T-\Delta} p\left(x, t^{\prime}+k_{s} ; v^{*}\right) c_{s}\left(x, t^{\prime}+k_{s}\right) \times\left[y\left(x, t^{\prime} ; v\right)-y\left(x, t^{\prime} ; v^{*}\right)\right] d t^{\prime} d \Gamma d k_{s} \\
& +\sum_{s=1}^{l} \int_{c_{s}}^{d_{s}} \int_{\Gamma} \int_{T-\Delta}^{T-k_{s}} p\left(x, t^{\prime}+k_{s} ; v^{*}\right) c_{s}\left(x, t^{\prime}+k_{s}\right) \times\left[y\left(x, t^{\prime} ; v\right)-y\left(x, t^{\prime} ; v^{*}\right)\right] d t^{\prime} d \Gamma d k_{s} \\
& +\int_{0}^{T} \int_{\Gamma} p\left(v^{*}\right)\left(v-v^{*}\right) d \Gamma d t \\
& =\sum_{s=1}^{l} \int_{c_{s}}^{d_{s}} \int_{\Gamma} \int_{-k_{s}}^{0} p\left(x, t^{\prime}+k_{s} ; v^{*}\right) c_{s}\left(x, t^{\prime}+k_{s}\right) \times\left[y\left(x, t^{\prime} ; v\right)-y\left(x, t^{\prime} ; v^{*}\right)\right] d t^{\prime} d \Gamma d k_{s} \\
& +\sum_{s=1}^{l} \int_{c_{s}}^{d_{s}} \int_{\Gamma} \int_{0}^{T-\Delta} p\left(x, t^{\prime}+k_{s} ; v^{*}\right) c_{s}\left(x, t^{\prime}+k_{s}\right) \times\left[y\left(x, t^{\prime} ; v\right)-y\left(x, t^{\prime} ; v^{*}\right)\right] d t^{\prime} d \Gamma d k_{s}
\end{aligned}
$$


Journal of Applied Mathematics

$$
\begin{aligned}
& +\sum_{s=1}^{l} \int_{c_{s}}^{d_{s}} \int_{\Gamma} \int_{T-\Delta+k_{s}}^{T} p\left(x, t ; v^{*}\right) c_{S}(x, t) \times\left[y\left(x, t-k_{s} ; v\right)-y\left(x, t-k_{s} ; v^{*}\right)\right] d t d \Gamma d k_{s} \\
& +\int_{0}^{T} \int_{\Gamma} p\left(v^{*}\right)\left(v-v^{*}\right) d \Gamma d t
\end{aligned}
$$

The last component in (4.15) can be rewritten as

$$
\begin{aligned}
\int_{0}^{T} \int_{\Gamma} & \frac{\partial p\left(v^{*}\right)}{\partial v_{\mathcal{A}^{*}}}\left[y(v)-y\left(v^{*}\right)\right] d \Gamma d t \\
\quad & \int_{0}^{T-\Delta} \int_{\Gamma} \frac{\partial p\left(v^{*}\right)}{\partial v_{\mathscr{A}^{*}}}\left[y(v)-y\left(v^{*}\right)\right] d \Gamma d t+\int_{T-\Delta}^{T} \int_{\Gamma} \frac{\partial p\left(v^{*}\right)}{\partial v_{\mathcal{A}^{*}}}\left[y(v)-y\left(v^{*}\right)\right] d \Gamma d t .
\end{aligned}
$$

Substituting (4.16) and (4.17) into (4.15) and then the results into (4.13), we obtain

$$
\begin{aligned}
\lambda_{1} \int_{Q} & \left(y\left(v^{*}\right)-z_{d}\right)\left[y(v)-y\left(v^{*}\right)\right] d x d t \\
= & \int_{0}^{T} \int_{\Gamma} p\left(v^{*}\right)\left(v-v^{*}\right) d \Gamma d t-\int_{Q} p\left(v^{*}\right) A(t)\left[y(v)-y\left(v^{*}\right)\right] d x d t \\
& -\sum_{i=1}^{m} \int_{a_{i}}^{b_{i}} \int_{\Omega} \int_{-h_{i}}^{0} b_{i}\left(x, t+h_{i}\right) p\left(x, t+h_{i} ; v^{*}\right) \times\left[y(x, t ; v)-y\left(x, t ; v^{*}\right)\right] d t d x d h_{i} \\
& -\sum_{i=1}^{m} \int_{a_{i}}^{b_{i}} \int_{\Omega} \int_{0}^{T-\Delta} b_{i}\left(x, t+h_{i}\right) p\left(x, t+h_{i} ; v^{*}\right) \times\left[y(x, t ; v)-y\left(x, t ; v^{*}\right)\right] d t d x d h_{i} \\
& +\int_{Q} p\left(v^{*}\right) A(t)\left[y(v)-y\left(v^{*}\right)\right] d x d t \\
& -\sum_{i=1}^{m} \int_{a_{i}}^{b_{i}} \int_{\Omega} \int_{T-\Delta+h_{i}}^{T} p\left(x, t ; v^{*}\right) b_{i}(x, t) \times\left[y\left(x, t-h_{i} ; v\right)-y\left(x, t-h_{i} ; v^{*}\right)\right] d t d x d h_{i} \\
& +\sum_{s=1}^{l} \int_{\mathcal{C}_{s}}^{d_{s}} \int_{\Gamma} \int_{-k_{s}}^{0} c_{s}\left(x, t+k_{s}\right) p\left(x, t+k_{s} ; v^{*}\right) \times\left[y(x, t ; v)-y\left(x, t ; v^{*}\right)\right] d t d \Gamma d k_{s} \\
& -\sum_{s=1}^{l} \int_{\mathcal{C}_{s}}^{d_{s}} \int_{\Gamma} \int_{0}^{T-\Delta} c_{S}\left(x, t+k_{s}\right) p\left(x, t+k_{s} ; v^{*}\right) \times\left[y(x, t ; v)-y\left(x, t ; v^{*}\right)\right] d t d \Gamma d k_{s} \\
& -\sum_{s=1}^{l} \int_{\mathcal{C}_{s}}^{d_{s}} \int_{\Gamma} \int_{T-\Delta+k_{s}}^{T} c_{s}(x, t) p\left(x, t ; v^{*}\right) \times\left[y\left(x, t-k_{s} ; v\right)-y\left(x, t-k_{s} ; v^{*}\right)\right] d t d \Gamma d k_{s}
\end{aligned}
$$




$$
\begin{aligned}
& -\int_{\Gamma} \int_{0}^{T-\Delta} \frac{\partial p\left(v^{*}\right)}{\partial v_{\AA^{*}}} \times\left[y(x, t ; v)-y\left(x, t ; v^{*}\right)\right] d t d \Gamma \\
& -\int_{\Gamma} \int_{T-\Delta}^{T} \frac{\partial p\left(v^{*}\right)}{\partial v_{A^{*}}} \times\left[y(x, t ; v)-y\left(x, t ; v^{*}\right)\right] d t d \Gamma \\
& +\sum_{i=1}^{m} \int_{a_{i}}^{b_{i}} \int_{\Omega} \int_{0}^{T-\Delta} b_{i}\left(x, t+h_{i}\right) p\left(x, t+h_{i} ; v^{*}\right) \times\left[y(x, t ; v)-y\left(x, t ; v^{*}\right)\right] d t d x d h_{i} .
\end{aligned}
$$

Afterwards, using the facts that $y(x, t, v)=y\left(x, t, v^{*}\right)=\Phi_{0}(x, t)$ for $x \in \Omega$ and $t \in$ $[-\Delta, 0)$ and $y(x, t, v)=y\left(x, t, v^{*}\right)=\Psi_{0}(x, t)$ for $x \in \Gamma$ and $t \in[-\Delta, 0),\left.p\right|_{\Omega}\left(x, t ; v^{*}\right)=0$ and consequently $\left.p\right|_{\Gamma}\left(x, t ; v^{*}\right)=0$ for $t \in[T-\Delta+\lambda, T)$, we obtain

$$
\lambda_{1} \int_{Q}\left[y\left(v^{*}\right)-z_{d}\right] \times\left(y(v)-y\left(v^{*}\right)\right) d x d t=\int_{0}^{T} \int_{\Gamma} p\left(v^{*}\right)\left(v-v^{*}\right) d \Gamma d t
$$

Substituting (4.19) into (4.5) gives

$$
\int_{0}^{T} \int_{\Gamma}\left(p\left(v^{*}\right)+\lambda_{2} N v^{*}\right)\left(v-v^{*}\right) d \Gamma d t \geq 0, \quad \forall v \in U_{\mathrm{ad}}
$$

The foregoing result is now summarized.

Theorem 4.2. For the problem (3.1)-(3.5), with the performance functional (4.1) with $z_{d} \in L^{2}(Q)$ and $\lambda_{2}>0$ and with constraints on controls (4.2), there exists a unique optimal control $v^{*}$ which satisfies the maximum condition (4.20).

\subsection{Mathematical Examples}

Example 4.3. Consider now the particular case where $U_{\mathrm{ad}}=U=L^{2}(\Sigma)$ (no constraints case). Thus the maximum condition (4.20) is satisfied when

$$
v^{*}=-\lambda_{2} N^{-1} p\left(v^{*}\right)
$$

If $N$ is the identity operator on $L^{2}(\Sigma)$, then from Lemma 4.1 it follows that $v^{*} \in W^{\infty, 1}(Q)$.

Example 4.4. We can also consider an analogous optimal control problem where the performance functional is given by:

$$
I(v)=\lambda_{1} \int_{\Sigma}\left[\left.y(x, t ; v)\right|_{\Sigma}-z_{d}\right]^{2} d \Gamma d t+\lambda_{2} \int_{\Sigma}(N v) v d \Gamma d t
$$

where $z_{d} \in L^{2}(\Sigma)$. 
From Theorem 3.4 and the Trace Theorem [20, Vol. 2, page 9], for each $v \in L^{2}(\Sigma)$, there exists a unique solution $y(v) \in W^{\infty, 1}(Q)$ with $\left.y\right|_{\Sigma} \in L^{2}(\Sigma)$. Thus, $I(v)$ is well defined. Then, the optimal control $v^{*}$ is characterized by:

$$
\lambda_{1} \int_{\Sigma}\left(\left.y\left(v^{*}\right)\right|_{\Sigma}-z_{d}\right)\left[\left.y(v)\right|_{\Sigma}-\left.y\left(v^{*}\right)\right|_{\Sigma}\right] d \Gamma d t+\lambda_{2} \int_{\Sigma} N v^{*}\left(v-v^{*}\right) d \Gamma d t \geq 0, \quad \forall v \in U_{\mathrm{ad}}
$$

We define the adjoint variable $p=p\left(v^{*}\right)=p\left(x, t ; v^{*}\right)$ as the solution of the equations:

$$
\begin{gathered}
-\frac{\partial p\left(v^{*}\right)}{\partial t}+\mathcal{A}^{*}(t) p\left(v^{*}\right)+\sum_{i=1}^{m} \int_{a_{i}}^{b_{i}} b_{i}\left(x, t+h_{i}\right) p\left(x, t+h_{i} ; v^{*}\right) d h_{i}=0, \\
(x, t) \in \Omega \times(0, T-\Delta), \quad h_{i} \in\left(a_{i}, b_{i}\right), \\
\frac{-\partial p\left(v^{*}\right)}{\partial t}+\mathcal{A}^{*}(t) p\left(v^{*}\right)=0, \quad(x, t) \in \Omega \times(T-\Delta, T), \\
p\left(x, T ; v^{*}\right)=0, \quad x \in \Omega, \\
p\left(x, t ; v^{*}\right)=0, \quad(x, t) \in \Omega \times[T-\Delta+\lambda, T), \\
\frac{\partial p\left(v^{*}\right)}{\partial v_{\mathcal{A}^{*}}}(x, t)=\sum_{s=1}^{l} \int_{\mathcal{c}_{S}}^{d_{S}} c_{S}\left(x, t+k_{s}\right) p\left(x, t+k_{s} ; v^{*}\right) d k_{s}+\lambda_{1}\left(\left.y v^{*}\right|_{\Sigma}-z_{\Sigma d}\right), \\
\quad(x, t) \in \Gamma \times(0, T-\Delta(T)), \quad k_{s} \in\left(c_{s}, d_{S}\right), \\
\frac{\partial p\left(v^{*}\right)}{\partial v_{\mathcal{A}^{*}}}(x, t)=\lambda_{1}\left(\left.y\left(v^{*}\right)\right|_{\Sigma}-z_{\Sigma d}\right), \quad(x, t) \in \Gamma \times(T-\Delta(T), T) .
\end{gathered}
$$

As in the above section, we have the following result.

Lemma 4.5. Let the hypothesis of Theorem 3.4 be satisfied. Then, for given $z_{\Sigma d} \in L^{2}(\Sigma)$ and any $v \in L^{2}(\Sigma)$, there exists a unique solution $p\left(v^{*}\right) \in W^{\infty, 1}(Q)$ to the adjoint problem (4.24).

Using the adjoint equations (4.24)in this case, the condition (4.23) can also be written in the following form:

$$
\int_{0}^{T} \int_{\Gamma}\left(p\left(v^{*}\right)+\lambda_{2} N v^{*}\right)\left(v-v^{*}\right) d \Gamma d t \geq 0, \quad \forall v \in U_{\mathrm{ad}}
$$

The following result is now summarized.

Theorem 4.6. For the problem (3.1)-(3.5) with the performance function (4.22) with $z_{\Sigma d} \in L^{2}(\Sigma)$ and $\lambda_{2}>0$, and with constraint (4.2), and with adjoint equations (4.24), there exists a unique optimal control $v^{*}$ which satisfies the maximum condition (4.25). 
Example $4.7\left(u \in L^{2}(Q)\right)$. We can also consider an analogous optimal control problem where the performance functional is given by:

$$
I(u)=\lambda_{1} \int_{Q}\left[y(x, t ; u)-z_{d}\right]^{2} d x d t+\lambda_{2} \int_{Q}(N u) u d x d t
$$

where $z_{d} \in L^{2}(Q)$.

From Theorem 3.4 and the Trace Theorem [20, Vol. 2, page 9], for each $u \in L^{2}(Q)$, there exists a unique solution $y(u) \in W^{\infty, 1}(Q)$. Thus, $I$ is well defined. Then, the optimal control $u^{*}$ is characterized by:

$$
\lambda_{1} \int_{Q}\left(y\left(u^{*}\right)-z_{d}\right)\left[y(u)-y\left(u^{*}\right)\right] d x d t+\lambda_{2} \int_{Q} N u^{*}\left(u-u^{*}\right) d x d t \geq 0, \quad \forall u \in U_{\mathrm{ad}}
$$

We define the adjoint variable $p=p\left(u^{*}\right)=p\left(x, t ; u^{*}\right)$ as the solution of the equations:

$$
\begin{gathered}
-\frac{\partial p\left(u^{*}\right)}{\partial t}+\mathcal{A}^{*}(t) p\left(u^{*}\right)+\sum_{i=1}^{m} \int_{a_{i}}^{b_{i}} b_{i}\left(x, t+h_{i}\right) p\left(x, t+h_{i} ; u^{*}\right) d h_{i}=\lambda_{1}\left(y\left(u^{*}\right)-z_{d}\right), \\
(x, t) \in \Omega \times(0, T-\Delta), \quad h_{i} \in\left(a_{i}, b_{i}\right), \\
-\frac{\partial p\left(u^{*}\right)}{\partial t}+\mathcal{A}^{*}(t) p\left(u^{*}\right)=\lambda_{1}\left(y\left(u^{*}\right)-z_{d}\right), \quad(x, t) \in \Omega \times(T-\Delta, T), \\
p\left(x, T ; u^{*}\right)=0, \quad x \in \Omega, \\
p\left(x, t ; u^{*}\right)=0, \quad(x, t) \in \Omega \times[T-\Delta+\lambda, T), \\
\frac{\partial p\left(u^{*}\right)}{\partial v_{\mathcal{A}^{*}}}(x, t)=\sum_{s=1}^{l} \int_{c_{s}}^{d_{s}} c_{S}\left(x, t+k_{s}\right) p\left(x, t+k_{s} ; u^{*}\right) d k_{s}, \\
(x, t) \in \Gamma \times(0, T-\Delta(T)), \quad k_{s} \in\left(c_{s}, d_{s}\right), \\
\frac{\partial p\left(u^{*}\right)}{\partial v_{A^{*}}}(x, t)=0, \quad(x, t) \in \Gamma \times(T-\Delta(T), T) .
\end{gathered}
$$

As in the above section, we have the following result.

Lemma 4.8. Let the hypothesis of Theorem 3.4 be satisfied. Then, for given $z_{d} \in L^{2}(Q)$ and any $u \in L^{2}(Q)$, there exists a unique solution $p\left(u^{*}\right) \in W^{\infty, 1}(Q)$ to the adjoint problem (4.28).

Using the adjoint equations (4.28) in this case, the condition (4.27) can also be written in the following form:

$$
\int_{Q}\left(p\left(u^{*}\right)+\lambda_{2} N u^{*}\right)\left(u-u^{*}\right) d x d t \geq 0, \quad \forall u \in U_{a d}
$$

The following result is now summarized. 
Theorem 4.9. For the problem (3.1)-(3.5) with the performance function (4.26) with $z_{d} \in L^{2}(Q)$ and $\lambda_{2}>0$, and with constraint (4.2), and with adjoint equations (4.28), there exists a unique optimal control $u^{*}$ which satisfies the maximum condition (4.29).

Example 4.10. We can also consider an analogous optimal control problem where the performance functional is given by:

$$
I(u)=\lambda_{1} \int_{\Sigma}\left[\left.y\right|_{\Sigma}(x, t ; u)-z_{\Sigma}\right]^{2} d \Gamma d t+\lambda_{2} \int_{Q}(N u) u d x d t
$$

where $z_{\Sigma d} \in L^{2}(\Sigma)$.

From Theorem 3.4 and the Trace Theorem [20, Vol. 2, page 9], for each $u \in L^{2}(Q)$, there exists a unique solution $y(u) \in W^{\infty, 1}(Q)$ with $\left.y\right|_{\Sigma} \in L^{2}(\Sigma)$. Thus, $I$ is well defined. Then, the optimal control $u^{*}$ is characterized by:

$$
\lambda_{1} \int_{\Sigma}\left(y\left(u^{*}\right)-z_{\Sigma d}\right)\left[y(u)-y\left(u^{*}\right)\right] d \Gamma d t+\lambda_{2} \int_{Q} N u^{*}\left(u-u^{*}\right) d x d t \geq 0, \quad \forall u \in U_{\mathrm{ad}}
$$

The above inequality can be simplified by introducing an adjoint equation, the form of which is identical to (4.24). Then using Theorem 3.4 we can establish the existence of a unique solution $p=p\left(u^{*}\right)=p\left(x, t ; u^{*}\right) \in W^{\infty, 1}(Q)$ for $(4.24)$.

As in the above section, we have the following result.

Lemma 4.11. Let the hypothesis of Theorem 3.4 be satisfied. Then, for given $z_{\Sigma d} \in L^{2}(\Sigma)$ and any $u \in L^{2}(Q)$, there exists a unique solution $p\left(u^{*}\right) \in W^{\infty, 1}(Q)$ to the adjoint problem (4.24)-(37).

Using the adjoint equations (4.24)-(37) in this case, the condition (4.31) can also be written in the following form:

$$
\int_{Q}\left(p\left(u^{*}\right)+\lambda_{2} N u^{*}\right)\left(u-u^{*}\right) d x d t \geq 0, \quad \forall u \in U_{a d}
$$

The following result is now summarized.

Theorem 4.12. For the problem (3.1)-(3.5) with the performance function (4.30) with $z_{\Sigma d} \in L^{2}(\Sigma)$ and $\lambda_{2}>0$, and with constraint (4.2), and with adjoint equations (4.24), there exists a unique optimal control $u^{*}$ which satisfies the maximum condition (4.32).

\section{Generalization}

The optimal control problems presented here can be extended to certain different two cases. Case 1: optimal control for $2 \times 2$ coupled infinite order parabolic systems with multiple time delays. Case 2: optimal control for $n \times n$ coupled infinite order parabolic systems with multiple time delays. Such extension can be applied to solving many control problems in mechanical engineering. 
Case 1 (optimal control for $2 \times 2$ coupled infinite order parabolic systems with multiple time delays). We can extend the discussions to study the optimal control for $2 \times 2$ coupled infinite order parabolic systems with multiple time delays. We consider the case where $v=\left(v_{1}, v_{2}\right) \in$ $L^{2}(\Sigma) \times L^{2}(\Sigma)$, the performance functional is given by $[15,16]$ :

$$
I(v)=I_{1}(v)+I_{2}(v)=\sum_{i=1}^{2}\left(\lambda_{1} \int_{Q}\left[y_{i}(x, t ; v)-z_{i d}\right]^{2} d x d t+\lambda_{2} \int_{\Sigma}\left(N_{i} v_{i}\right) v_{i} d x d t\right)
$$

where $z_{d}=\left(z_{1 d}, z_{2 d}\right) \in\left(L^{2}(Q)\right)^{2}$.

The following results can now be proved.

Theorem 5.1. Let $y_{0}, \Phi_{0}, \Psi_{0}, v$, and $u$ be given with $y_{0}=\left(y_{0,1}, y_{0,2}\right) \in\left(W^{\infty}\left\{\alpha_{\alpha}, 2\right\}(\Omega)\right)^{2}, \Psi_{0}=$ $\left(\Psi_{0,1}, \Psi_{0,2}\right) \in\left(L^{2}\left(\Sigma_{0}\right)\right)^{2}, \Phi_{0}=\left(\Phi_{0,1}, \Phi_{0,2}\right) \in\left(W^{\infty, 1}\left(Q_{0}\right)\right)^{2}, v=\left(v_{1}, v_{2}\right) \in\left(L^{2}(\Sigma)\right)^{2}$, and $u=$ $\left(u_{1}, u_{2}\right) \in\left(W^{-\infty,-1}(Q)\right)^{2}$. Then, there exists a unique solution $y=\left(y_{1}, y_{2}\right) \in\left(W^{-\infty, 1}(Q)\right)^{2}$ for the following mixed initial-boundary value problem:

$$
\begin{aligned}
& \frac{\partial y_{1}}{\partial t}+\left(\sum_{|\alpha|=0}^{\infty}(-1)^{|\alpha|} a_{\alpha} D^{2 \alpha}+1\right) y_{1}+\sum_{i=1}^{m} \int_{a_{i}}^{b_{i}} b_{i}(x, t) y_{1}\left(x, t-h_{i}\right) d h_{i}-y_{2}=u_{1}, \\
& \text { in } Q, h_{i} \in\left(a_{i}, b_{i}\right) \text {, } \\
& \frac{\partial y_{2}}{\partial t}+\left(\sum_{|\alpha|=0}^{\infty}(-1)^{|\alpha|} a_{\alpha} D^{2 \alpha}+1\right) y_{2}+\sum_{i=1}^{m} \int_{a_{i}}^{b_{i}} b_{i}(x, t) y_{2}\left(x, t-h_{i}\right) d h_{i}+y_{1}=u_{2} \\
& \text { in } Q, h_{i} \in\left(a_{i}, b_{i}\right) \text {, } \\
& y_{1}\left(x, t^{\prime} ; u\right)=\Phi_{0,1}\left(x, t^{\prime}\right), \quad\left(x, t^{\prime}\right) \in \Omega \times[-\Delta, 0), \\
& y_{2}\left(x, t^{\prime} ; u\right)=\Phi_{0,2}\left(x, t^{\prime}\right), \quad\left(x, t^{\prime}\right) \in \Omega \times[-\Delta, 0), \\
& y_{1}(x, 0 ; u)=y_{0,1}, \quad x \in \Omega, \\
& y_{2}(x, 0 ; u)=y_{0,2}, \quad x \in \Omega, \\
& \frac{\partial y_{1}}{\partial v_{A}}(x, t)=\sum_{s=1}^{l} \int_{c_{s}}^{d_{s}} c_{s 1}(x, t) y_{1}\left(x, t-k_{s}\right) d k_{s}+v_{1}, \quad \text { on } \Sigma, k_{s} \in\left(c_{s}, d_{s}\right), \\
& \frac{\partial y_{2}}{\partial v_{A}}=\sum_{s=1}^{l} \int_{c_{s}}^{d_{s}} c_{s 2}(x, t) y_{2}\left(x, t-k_{s}\right) d k_{s}+v_{2}, \quad \text { on } \Sigma, k_{s} \in\left(c_{s}, d_{s}\right), \\
& y_{1}\left(x, t^{\prime} ; u\right)=\Psi_{0,1}\left(x, t^{\prime}\right), \quad\left(x, t^{\prime}\right) \in \Gamma \times[-\Delta, 0), \\
& y_{2}\left(x, t^{\prime} ; u\right)=\Psi_{0,2}\left(x, t^{\prime}\right), \quad\left(x, t^{\prime}\right) \in \Gamma \times[-\Delta, 0),
\end{aligned}
$$


where

$$
\begin{gathered}
y \equiv y(x, t ; u)=\left(y_{1}(x, t ; u), y_{2}(x, t ; u)\right) \in\left(W^{\infty, 1}(Q)\right)^{2}, \\
u \equiv u(x, t)=\left(u_{1}(x, t), u_{2}(x, t)\right) \in\left(\left(W^{\infty, 1}(Q)\right)^{\prime}\right)^{2} \\
v \equiv v(x, t)=\left(v_{1}(x, t), v_{2}(x, t)\right) \in\left(L^{2}(\Sigma)\right)^{2}
\end{gathered}
$$

Lemma 5.2. Let the hypothesis of Theorem 5.1 be satisfied. Then for given $z_{d}=\left(z_{1 d}, z_{2 d}\right) \in\left(L^{2}(Q)\right)^{2}$ and any $v=\left(v_{1}, v_{2}\right) \in\left(L^{2}(\Sigma)\right)^{2}$, there exists a unique solution $p(v)=\left(p_{1}(v), p_{2}(v)\right) \in\left(W^{\infty, 1}(Q)\right)^{2}$ for the adjoint problem:

$$
\begin{aligned}
& -\frac{\partial p_{1}(v)}{\partial t}+\left(\sum_{|\alpha|=0}^{\infty}(-1)^{|\alpha|} a_{\alpha} D^{2 \alpha}+1\right) p_{1}(v)+\sum_{i=1}^{m} \int_{a_{i}}^{b_{i}} b_{i}\left(x, t+h_{i}\right) p_{1}\left(x, t+h_{i} ; v\right) d h_{i}+p_{2}(v) \\
& =\lambda_{1}\left(y_{1}(v)-z_{1 d}\right), \quad(x, t) \in \Omega \times(0, T-\Delta), h_{i} \in\left(a_{i}, b_{i}\right), \\
& -\frac{\partial p_{2}(v)}{\partial t}+\left(\sum_{|\alpha|=0}^{\infty}(-1)^{|\alpha|} a_{\alpha} D^{2 \alpha}+1\right) p_{2}(v)+\sum_{i=1}^{m} \int_{a_{i}}^{b_{i}} b_{i}\left(x, t+h_{i}\right) p_{2}\left(x, t+h_{i} ; v\right) d h_{i}-p_{1}(v) \\
& =\lambda_{1}\left(y_{2}(v)-z_{2 d}\right), \quad(x, t) \in \Omega \times(0, T-\Delta), h_{i} \in\left(a_{i}, b_{i}\right), \\
& \frac{\partial p_{1}(v)}{\partial t}+\left(\sum_{|\alpha|=0}^{\infty}(-1)^{|\alpha|} a_{\alpha} D^{2 \alpha}+1\right) p_{1}(v)=\lambda_{1}\left(y_{1}(v)-z_{1 d}\right), \quad(x, t) \in \Omega \times(T-\Delta, T), \\
& \frac{\partial p_{2}(v)}{\partial t}+\left(\sum_{|\alpha|=0}^{\infty}(-1)^{|\alpha|} a_{\alpha} D^{2 \alpha}+1\right) p_{2}(v)=\lambda_{1}\left(y_{2}(v)-z_{2 d}\right), \quad(x, t) \in \Omega \times(T-\Delta, T), \\
& p_{1}(x, T ; v)=0, \quad p_{2}(x, T ; v)=0, \quad x \in \Omega, \\
& p_{1}(x, t ; v)=0, \quad p_{2}(x, t ; v)=0, \quad(x, t) \in \Omega \times[T-\Delta+\lambda, T), \\
& \frac{\partial p_{1}(x, t ; v)}{\partial v_{\mathcal{A}^{*}}}=\sum_{s=1}^{l} \int_{\mathcal{C}_{s}}^{d_{s}} c_{s 1}\left(x, t+k_{s}\right) p_{1}\left(x, t+k_{s} ; v\right) d k_{s}, \quad(x, t) \in \Gamma \times(0, T-\Delta), k_{s} \in\left(c_{s}, d_{s}\right), \\
& \frac{\partial p_{2}(x, t ; v)}{\partial v_{A^{*}}}=\sum_{s=1}^{l} \int_{c_{s}}^{d_{s}} c_{s 2}\left(x, t+k_{s}\right) p_{2}\left(x, t+k_{s} ; v\right) d k_{s}, \quad(x, t) \in \Gamma \times(0, T-\Delta), k_{s} \in\left(c_{s}, d_{s}\right), \\
& \frac{\partial p_{1}(x, t)}{\partial v_{\mathbb{A}^{*}}}=0, \quad \frac{\partial p_{2}(x, t)}{\partial v_{\mathcal{A}^{*}}}=0, \quad(x, t) \in \Gamma \times(T-\Delta(T), T) .
\end{aligned}
$$


Theorem 5.3. The optimal control $v^{*} \equiv v^{*}(x, t)=\left(v_{1}^{*}(x, t), v_{2}^{*}(x, t)\right) \in\left(L^{2}(\Sigma)\right)^{2}$ is characterized by the following maximum condition:

$$
\begin{array}{r}
\int_{0}^{T} \int_{\Gamma}\left(\left[p_{1}\left(v^{*}\right)+\lambda_{2} N_{1} v_{1}^{*}\right]\left(v_{1}-v_{1}^{*}\right)+\left[p_{2}\left(v^{*}\right)+\lambda_{2} N_{2} v_{2}^{*}\right]\left(v_{2}-v_{2}^{*}\right)\right) d \Gamma d t \geq 0, \\
\forall v=\left(v_{1}, v_{2}\right) \in\left(L^{2}(\Sigma)\right)^{2}
\end{array}
$$

where $p \equiv p(x, t ; v)=\left(p_{1}(x, t ; v), p_{2}(x, t ; v)\right) \in\left(W^{\infty, 1}(Q)\right)^{2}$ is the adjoint state.

The foregoing result is now summarized.

Theorem 5.4. For the problem (5.2) with the performance function (5.1) with $z_{d}=\left(z_{1 d}, z_{2 d}\right) \in$ $\left(L^{2}(Q)\right)^{2}$ and $\lambda_{2}>0$, and with constraint: $U_{a d}$ is closed, convex subset of $\left(L^{2}(\Sigma)\right)^{2}$, and with adjoint problem (5.4), then there exists a unique optimal control $v^{*} \equiv v^{*}(x, t)=\left(v_{1}^{*}(x, t), v_{2}^{*}(x, t)\right) \in$ $\left(L^{2}(\Sigma)\right)^{2}$ which satisfies the maximum condition (5.5).

Case 2 (optimal control for $(n \times n)$ coupled infinite order parabolic systems with multiple time delays). We will extend the discussion to $(n \times n)$ coupled infinite order parabolic systems. We consider the case where $v=\left(v_{1}, v_{2}, \ldots, v_{n}\right) \in\left(\left(L^{2}(\Sigma)\right)^{n}\right.$, the performance functional is given by $[15,16]$ :

$$
I(v)=\sum_{j=1}^{n}\left(\lambda_{1} \int_{Q}\left[y_{j}(x, t ; v)-z_{j d}\right]^{2} d x d t+\lambda_{2} \int_{\Sigma}\left(N_{j} v_{j}\right) v_{j} d x d t\right)
$$

where $z_{d}=\left(z_{1 d}, z_{2 d}, \ldots, z_{n d}\right) \in\left(L^{2}(Q)\right)^{n}$.

The following results can now be proved.

Theorem 5.5. Let $y_{0}, \Phi_{0}, \Psi_{0}, v$, and $u$ be given with $y_{p}=\left(y_{p, 1}, y_{p, 2}, \ldots, y_{p, n}\right) \in\left(W^{\infty}\left\{a_{\alpha}, 2\right\}(\Omega)\right)^{n}$, $\Phi_{0}=\left(\Phi_{0,1}, \Phi_{0,2}, \ldots, \Phi_{0, n}\right) \in\left(W^{\infty, 1}\left(Q_{0}\right)\right)^{n}, \Psi_{0}=\left(\Psi_{0,1}, \Psi_{0,2}, \ldots, \Psi_{0, n}\right) \in\left(L^{2}\left(\Sigma_{0}\right)\right)^{n}, v=$ $\left(v_{1}, v_{2}, \ldots, v_{n}\right) \in\left(L^{2}\left(\Sigma_{0}\right)\right)^{n}$, and $u=\left(u_{1}, u_{2}, \ldots, u_{n}\right) \in\left(W^{-\infty,-1}(Q)\right)^{n}$. Then, there exists a unique solution $y=\left(y_{1}, y_{2}, \ldots, y_{n}\right) \in\left(W^{\infty, 1}\left(Q_{0}\right)\right)^{n}$ for the following mixed initial-boundary value problem: for all $j, j=1,2, \ldots, n$ one has

$$
\begin{aligned}
& \frac{\partial y_{j}}{\partial t}+\mathcal{S}(t) y_{j}(x, t)+\sum_{i=1}^{m} \int_{a_{i}}^{b_{i}} b_{i}(x, t) y_{j}\left(x, t-h_{i}\right) d h_{i}=u_{j}, \\
& \qquad(x, t) \in \Omega \times(0, T), \quad h_{i} \in\left(a_{i}, b_{i}\right), \\
& y_{j}\left(x, t^{\prime}\right)=\Phi_{0, j}\left(x, t^{\prime}\right) \quad\left(x, t^{\prime}\right) \in \Omega \times[-\Delta, 0), \\
& y_{j}(x, 0)=y_{0, j}(x), \quad x \in \Omega,
\end{aligned}
$$


Journal of Applied Mathematics

$$
\begin{gathered}
\frac{\partial y_{j}}{\partial v_{S}}(x, t)=\sum_{S=1}^{l} \int_{c_{s}}^{d_{s}} c_{S}(x, t) y_{j}\left(x, t-k_{s}\right) d k_{s}+v_{j}, \\
(x, t) \in \Gamma \times(0, T), \quad k_{s} \in\left(c_{s}, d_{s}\right), \\
y_{j}\left(x, t^{\prime}\right)=\Psi_{0, j}\left(x, t^{\prime}\right), \quad\left(x, t^{\prime}\right) \in \Gamma \times[-\Delta, 0),
\end{gathered}
$$

where

$$
\begin{gathered}
y \equiv y(x, t ; u)=\left(y_{1}(x, t ; u), y_{2}(x, t ; u), \ldots, y_{n}(x, t ; u)\right) \in\left(W^{\infty, 1}(Q)\right)^{n}, \\
u \equiv u(x, t)=\left(u_{1}(x, t), u_{2}(x, t), \ldots, u_{n}(x, t)\right) \in\left(W^{-\infty,-1}(Q)\right)^{n} \\
v \equiv v(x, t)=\left(v_{1}(x, t), v_{2}(x, t), \ldots, v_{n}(x, t)\right) \in\left(L^{2}(\Sigma)\right)^{n} .
\end{gathered}
$$

The operator $\mathcal{S}(t)$ is an $n \times n$ matrix takes the form $[15,16,18,22]$ :

$\mathcal{S}(t)$

$$
=\left(\begin{array}{cccc}
\sum_{|\alpha|=0}^{\infty}(-1)^{|\alpha|} a_{\alpha} D^{2 \alpha}+1 & -1 & \cdot & -1 \\
1 & \sum_{|\alpha|=0}^{\infty}(-1)^{|\alpha|} a_{\alpha} D^{2 \alpha}+1 & \cdot & -1 \\
\cdot & \cdot & \cdot & \cdot \\
\cdot & \cdot & \cdot & \cdot \\
1 & 1 & \cdot & \sum_{|\alpha|=0}^{\infty}(-1)^{|\alpha|} a_{\alpha} D^{2 \alpha}+1
\end{array}\right)_{n \times n},
$$

that is

$$
\mathcal{S}(t) y_{j}(x)=\sum_{|\alpha|=0}^{\infty}(-1)^{|\alpha|} a_{\alpha} D^{2 \alpha} y_{j}(x)+\sum_{r=1}^{n} B_{j r} y_{j}(x), \quad \forall j, j=1,2, \ldots, n,
$$

where

$$
B_{j r}= \begin{cases}1, & \text { if } j \geq r \\ -1, & \text { if } j<r\end{cases}
$$

Lemma 5.6. Let the hypothesis of Theorem 5.5 be satisfied. Then for given $z_{d}=\left(z_{1 d}, z_{2 d}, \ldots, z_{n d}\right) \in$ $\left(L^{2}(Q)\right)^{n}$ and any $v(x, t)=\left(v_{1}(x, t), v_{2}(x, t), \ldots, v_{n}(x, t)\right) \in\left(L^{2}(\Sigma)\right)^{n}$, there exists a unique solution

$$
p(v) \equiv p(x, t ; v)=\left(p_{1}(x, t ; v), p_{1}(x, t ; v), \ldots, p_{n}(x, t ; v)\right) \in\left(W^{\infty, 1}(Q)\right)^{n}
$$


for the adjoint problem: for all $j, j=1,2, \ldots, n$, one has

$$
\begin{gathered}
\frac{-\partial p_{j}(v)}{\partial t}+\mathcal{S}^{*}(t) p_{j}(v)+\sum_{i=1}^{m} \int_{a_{i}}^{b_{i}} b_{i}\left(x, t+h_{i}\right) p_{j}\left(x, t+h_{i} ; v\right) d h_{i}=\lambda_{1}\left(y_{j}(v)-z_{j d}\right), \\
(x, t) \in \Omega \times(0, T-\Delta), \quad h_{i} \in\left(a_{i}, b_{i}\right), \\
\frac{\partial p_{j}(v)}{\partial t}+\mathcal{S}^{*}(t) p_{j}(v)=\lambda_{j}\left(y_{j}(v)-z_{j d}\right), \quad(x, t) \in \Omega \times(T-\Delta, T), \\
p_{j}(x, T, v)=0, \quad x \in \Omega, \\
\frac{\partial p_{j}(v)}{\partial v_{\mathcal{S}^{*}}}(x, t)=\sum_{s=1}^{l} \int_{c_{s}}^{d_{s}} c_{S}\left(x, t+k_{s}\right) p_{j}\left(x, t+k_{s} ; v\right) d k_{s}, \quad(x, t) \in \Gamma \times(0, T-\Delta), \quad k_{s} \in\left(c_{s}, d_{s}\right), \\
\frac{\partial p_{j}(v)}{\partial v_{\mathcal{S}^{*}}}(x, t)=0, \quad(x, t) \in \Gamma \times(T-\Delta, T),
\end{gathered}
$$

where

$$
\mathcal{S}^{*}(t) p_{j}(x)=\sum_{|\alpha|=0}^{\infty}(-1)^{|\alpha|} a_{\alpha} D^{2 \alpha} p_{j}(x)+\sum_{r=1}^{n} B_{r j} p_{j}(x), \quad \forall j, j=1,2, \ldots, n
$$

$B_{r j}$ are the transpose of $B_{j r}$.

Theorem 5.7. The optimal control $v^{*} \equiv v^{*}(x, t)=\left(v_{1}^{*}(x, t), v_{2}^{*}(x, t), \ldots, v_{n}^{*}(x, t)\right) \in\left(L^{2}(\Sigma)\right)^{n}$ is characterized by the following maximum condition:

$$
\sum_{j=1}^{n} \int_{\Sigma}\left[p_{j}\left(v^{*}\right)+\lambda_{2} N_{j} v_{j}^{*}\right]\left(v_{j}-v_{j}^{*}\right) d \Gamma d t \geq 0, \quad \forall v=\left(v_{1}, v_{2}, \ldots, v_{n}\right) \in\left(U_{a d}\right)^{n}
$$

where

$$
p\left(v^{*}\right) \equiv p\left(x, t ; v^{*}\right)=\left(p_{1}\left(x, t ; v^{*}\right), p_{1}\left(x, t ; v^{*}\right), \ldots, p_{n}\left(x, t ; v^{*}\right)\right) \in\left(W^{\infty, 1}(Q)\right)^{n}
$$

is the adjoint state.

The foregoing result is now summarized.

Theorem 5.8. For the problem (5.7) with the performance function (5.6) with $z_{d}=$ $\left(z_{1 d}, z_{2 d}, \ldots, z_{n d}\right) \in\left(L^{2}(Q)\right)^{n}$ and $\lambda_{2}>0$, and with constraint: $U_{a d}$ is closed, convex subset of $\left(L^{2}(\Sigma)\right)^{n}$, and with adjoint equations (5.13), then there exists a unique optimal control $v^{*} \equiv v^{*}(x, t)=$ $\left(v_{1}^{*}(x, t), v_{2}^{*}(x, t), \ldots, v_{n}^{*}(x, t)\right) \in\left(L^{2}(\Sigma)\right)^{n}$ which satisfies the maximum condition (5.15). 
In the case of performance functionals (4.1), (4.22), (4.26), (4.30), (5.1), and (5.6) with $\lambda_{1}>0$ and $\lambda_{2}=0$, the optimal control problem reduces to minimization of the functional on a closed and convex subset in a Hilbert space. Then, the optimization problem is equivalent to a quadratic programming one, which can be solved by the use of the well-known Gilbert algorithm.

\section{Conclusions}

The optimization problem presented in the paper constitutes a generalization of the optimal boundary control problem for second-order parabolic systems with Neumann boundary condition involving constant time lag appearing in the state and in the boundary conditions considered in $[1,5-9,14-16,18,21,32]$.

Moreover, the results obtained in this paper (Theorems 4.2, 4.6, 5.4, and 5.8) can be treated as a generalization of the optimization theorems proved by [8-10]. Also the main result of the paper contains necessary and sufficient conditions of optimality for $(n \times n)$ infinite order parabolic systems with multiple time delays given in integral form both in the state equation and in the Neumann boundary condition that give characterization of optimal control (Theorem 5.8). But it is easily seen that obtaining analytical formulas for optimal control are very difficult. This results from the fact that state equations (5.7), adjoint equations (5.13), and maximum condition (5.15) are mutually connected that cause that the usage of derived conditions is difficult. Therefore we must resign from the exact determination of the optimal control and therefore we are forced to use approximation methods.

Also it is evident that by modifying:

(i) the boundary conditions, (Dirichlet, Neumann, mixed, etc.),

(ii) the nature of the control (distributed, boundary, etc.),

(iii) the nature of the observation (distributed, boundary, etc.),

(iv) the initial differential system,

(v) the time delays (constant time delays, time-varying delays, multiple time-varying delays, time delays given in the integral form, etc.),

(vi) the number of variables (finite number of variables, infinite number of variables systems, etc.),

(vii) the type of equation (elliptic, parabolic, hyperbolic, etc.),

(viii) the order of equation (second order, Schrödinger, infinite order, etc.),

(ix) the type of control ( optimal control problem, time-optimal control problem, etc.),

an infinity of variations on the above problems are possible to study with the help of [19] and Dubovitskii-Milyutin formalisms [22-27]. Those problems need further investigations and form tasks for future research. These ideas mentioned above will be developed in forthcoming papers.

\section{Acknowledgments}

The research presented here was carried out within the research programme of the Taibah University-Dean of Scientific Research under Project no. 806/1433. The author would like to express his gratitude to the anonymous reviewers for their very valuable remarks. 


\section{References}

[1] A. Kowalewski, "Optimization of parabolic systems with deviating arguments," International Journal of Control, vol. 72, no. 11, pp. 947-959, 1999.

[2] P. K. C. Wang, "Optimal control of parabolic systems with boundary conditions involving time delays," SIAM Journal on Control and Optimization, vol. 13, pp. 274-293, 1975.

[3] G. Knowles, "Time-optimal control of parabolic systems with boundary conditions involving time delays," Journal of Optimization Theory and Applications, vol. 25, no. 4, pp. 563-574, 1978.

[4] K. H. Wong, "Optimal control computation for parabolic systems with boundary conditions involving time delays," Journal of Optimization Theory and Applications, vol. 53, no. 3, pp. 475-507, 1987.

[5] A. Kowalewski, "Boundary control of distributed parabolic system with boundary condition involving a time-varying lag," International Journal of Control, vol. 48, no. 6, pp. 2233-2248, 1988.

[6] A. Kowalewski, "Optimal control of distributed parabolic systems involving time lags," IMA Journal of Mathematical Control and Information, vol. 7, no. 4, pp. 375-393, 1990.

[7] A. Kowalewski, "Optimal control of distributed parabolic systems with multiple time-varying lags," International Journal of Control, vol. 69, no. 3, pp. 361-381, 1998.

[8] A. Kowalewski, "Optimal control of distributed parabolic systems with multiple time delays given in the integral form," IMA Journal of Mathematical Control and Information, vol. 22, no. 2, pp. 149-170, 2005.

[9] A. Kowalewski, "Time-optimal control of infinite order hyperbolic systems with time delays," International Journal of Applied Mathematics and Computer Science, vol. 19, no. 4, pp. 597-608, 2009.

[10] A. Kowalewski and J. T. Duda, "An optimization problem for time lag distributed parabolic systems," IMA Journal of Mathematical Control and Information, vol. 21, no. 1, pp. 15-31, 2004.

[11] A. Kowalewski and A. Krakowiak, "Time-optimal boundary control of an infinite order parabolic system with time lags," International Journal of Applied Mathematics and Computer Science, vol. 18, no. 2, pp. 189-198, 2008.

[12] W. Kotarski, Some Problems of Optimal and Pareto Optimal Control for Distributed Parameter Systems, vol. 1668, Silesian University, Katowice, Poland, 1997.

[13] W. Kotarski, H. A. El-Saify, and G. M. Bahaa, “Optimal control of parabolic equation with an infinite number of variables for non-standard functional and time delay," IMA Journal of Mathematical Control and Information, vol. 19, no. 4, pp. 461-476, 2002.

[14] W. Kotarski and G. M. Bahaa, "Optimality conditions for infinite order hyperbolic control problem with non-standard functional and time delay," Journal of Information E Optimization Sciences, vol. 28, no. 3, pp. 315-334, 2007.

[15] H. A. El-Saify, "Optimal control of $n \times n$ parabolic system involving time lag," IMA Journal of Mathematical Control and Information, vol. 22, no. 3, pp. 240-250, 2005.

[16] H. A. El-Saify, "Optimal boundary control problem for $n \times n$ infinite-order parabolic lag system," IMA Journal of Mathematical Control and Information, vol. 23, no. 4, pp. 433-445, 2006.

[17] I. M. Gali, H. A. El-Saify, and S. A. El-Zahaby, "Distributed control of a system governed by Dirichlet and Neumann problems for elliptic equations of infinite order," in Functional-Differential Systems and Related Topics, III, pp. 83-87, Higher College Engineering, Zielona Góra, Poland, 1983.

[18] H. A. El-Saify and G. M. Bahaa, "Optimal control for $n \times n$ hyperbolic systems involving operators of infinite order," Mathematica Slovaca, vol. 52, no. 4, pp. 409-424, 2002.

[19] J.-L. Lions, Optimal Control of Systems Governed by Partial Differential Equations, Translated from the French by S. K. Mitter. Die Grundlehren der mathematischen Wissenschaften, Band 170, Springer, New York, NY, USA, 1971.

[20] J. L. Lions and E. Magenes, Non-Homogeneous Boundary Value Problem and Applications. I, Springer, New York, NY, USA, 1972

[21] W. Kotarski, H. A. El-Saify, and G. M. Bahaa, “Optimal control problem for a hyperbolic system with mixed control-state constraints involving operator of infinite order," International Journal of Pure and Applied Mathematics, vol. 1, no. 3, pp. 241-254, 2002.

[22] G. M. Bahaa, "Quadratic Pareto optimal control of parabolic equation with state-control constraints and an infinite number of variables," IMA Journal of Mathematical Control and Information, vol. 20, no. 2, pp. 167-178, 2003.

[23] G. M. Bahaa, "Time-optimal control problem for parabolic equations with control constraints and infinite number of variables," IMA Journal of Mathematical Control and Information, vol. 22, no. 3, pp. 364-375, 2005. 
[24] G. M. Bahaa, “Time-optimal control problem for infinite order parabolic equations with control constraints," Differential Equations and Control Processes, no. 4, pp. 64-81, 2005.

[25] G. M. Bahaa, “Optimal control for cooperative parabolic systems governed by Schrödinger operator with control constraints," IMA Journal of Mathematical Control and Information, vol. 24, no. 1, pp. 1-12, 2007.

[26] G. M. Bahaa, "Quadratic Pareto optimal control for boundary infinite order parabolic equation with state-control constraints," Advanced Modeling and Optimization, vol. 9, no. 1, pp. 37-51, 2007.

[27] G. M. Bahaa, "Optimal control problems of parabolic equations with an infinite number of variables and with equality constraints," IMA Journal of Mathematical Control and Information, vol. 25, no. 1, pp. 37-48, 2008.

[28] G. M. Bahaa and W. Kotarski, "Optimality conditions for $n \times n$ infinite-order parabolic coupled systems with control constraints and general performance index," IMA Journal of Mathematical Control and Information, vol. 25, no. 1, pp. 49-57, 2008.

[29] Ju. A. Dubinski1,, "Sobolev spaces of infinite order, and the behavior of the solutions of certain boundary value problems when the order of the equation increases indefinitely," vol. 98, no. 2, pp. 163-184, 1975 (Russian).

[30] Ju. A. Dubinskiř, "Nontriviality of Sobolev spaces of infinite order in the case of the whole Euclidean space and the torus," vol. 100, no. 3, pp. 436-446, 1976 (Russian).

[31] J. A. Dubinskii, "Sobolev spaces of infinite order and differential equations. Mathematics and its applications," East European Series, vol. 3, p. 164, 1988.

[32] W. Kotarski and G. M. Bahaa, "Optimal control problem for infinite order hyperbolic system with mixed control-state constraints," European Journal of Control, vol. 11, no. 2, pp. 150-156, 2005. 


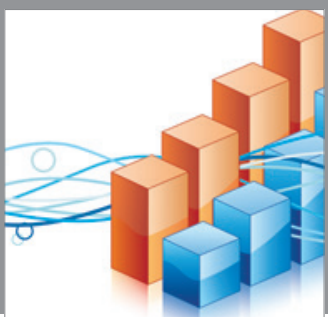

Advances in

Operations Research

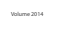

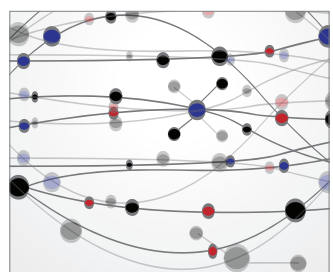

\section{The Scientific} World Journal
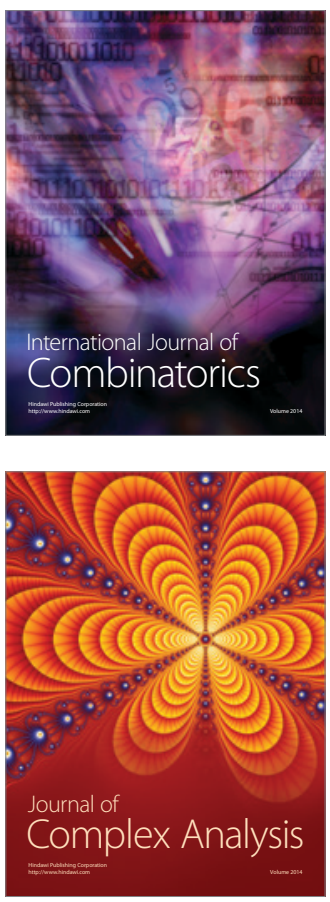

International Journal of

Mathematics and

Mathematical

Sciences
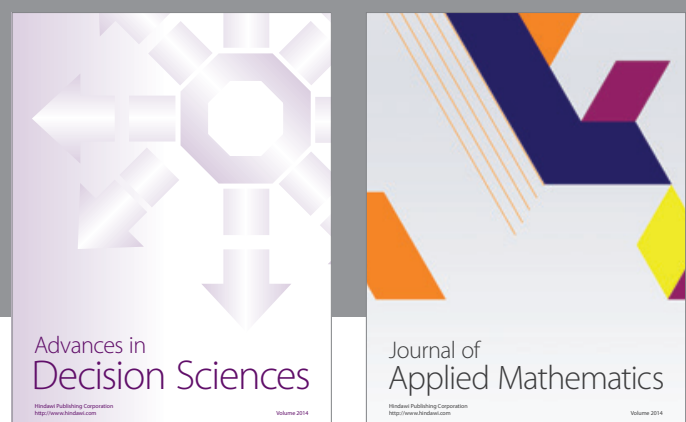

Journal of

Applied Mathematics
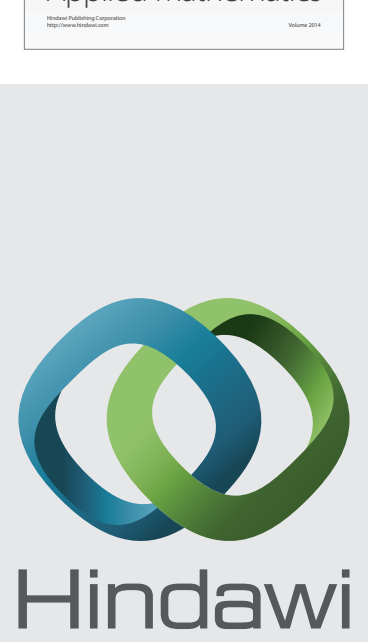

Submit your manuscripts at http://www.hindawi.com
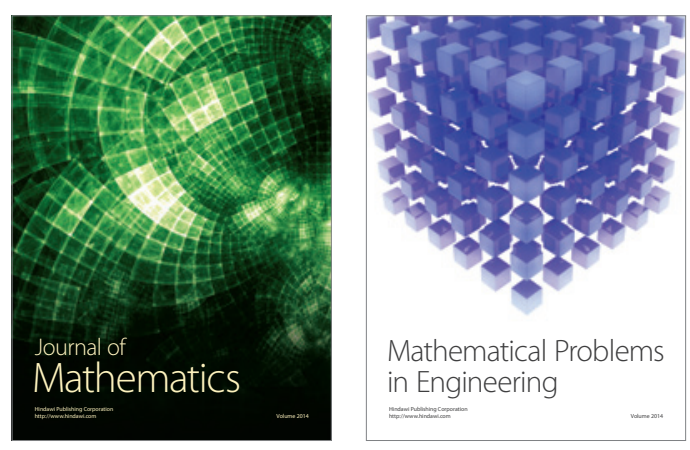

Mathematical Problems in Engineering
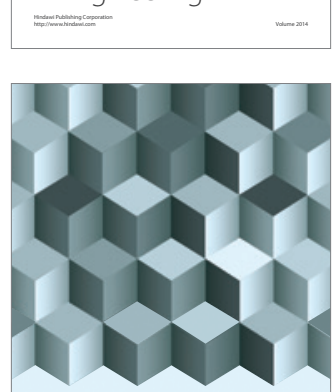

Journal of

Function Spaces
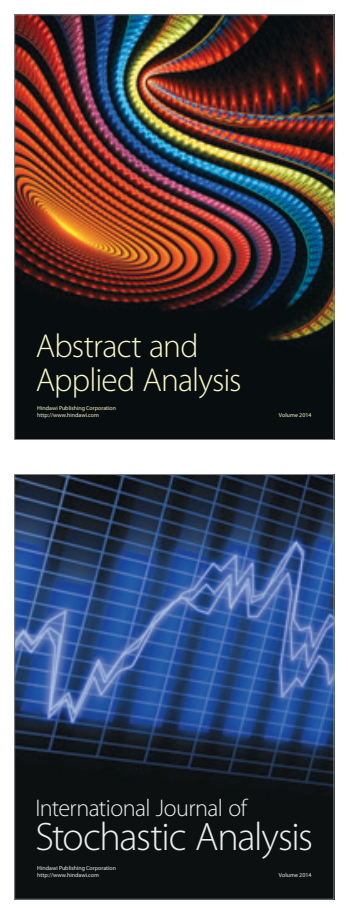

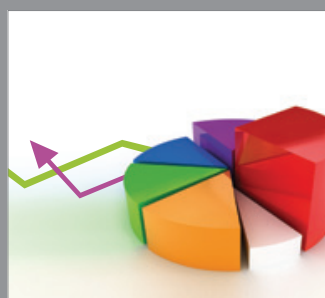

ournal of

Probability and Statistics

Promensencen
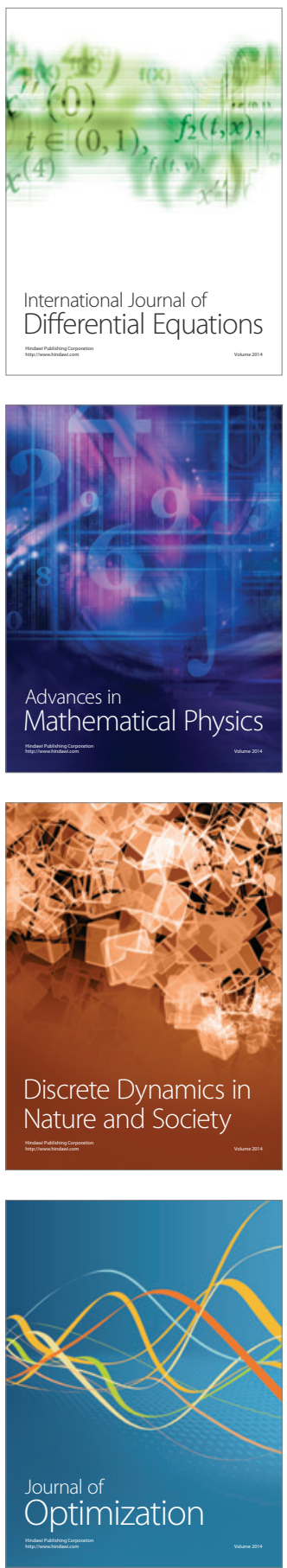